\title{
Explorer of light, and more: an interview with Sir Michael Berry
}

\author{
Wei Liu ${ }^{a, b, *}$ \\ anational University of Defense Technology, College of Advanced Interdisciplinary Studies, Changsha, China \\ ${ }^{b}$ National University of Defense Technology, Hunan Provincial Key Laboratory of Novel Nano-Optoelectronic Information Materials and \\ Devices, Changsha, China
}

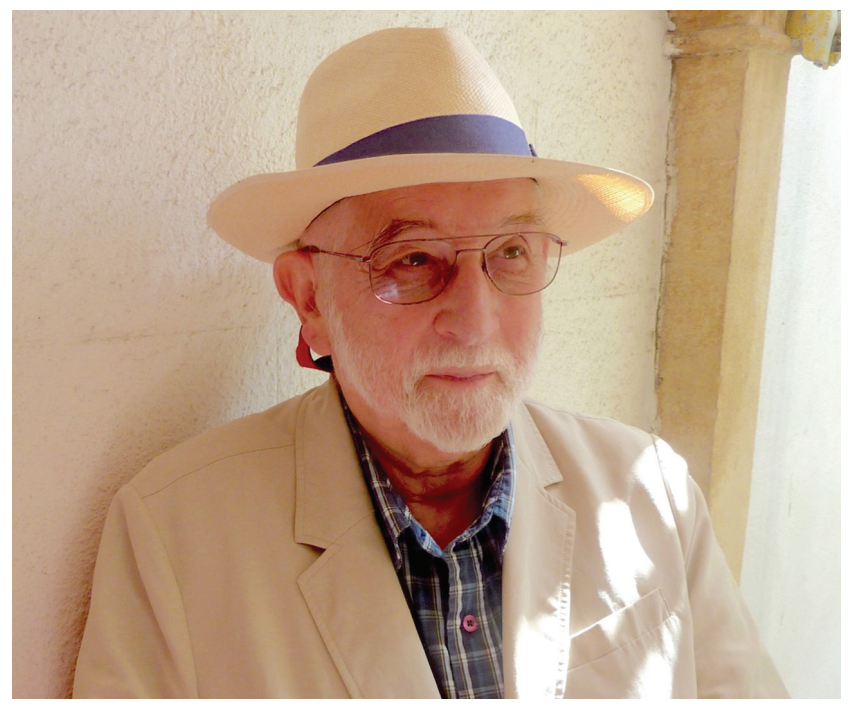

Sir Michael Berry, University of Bristol

Wei Liu: Professor Berry, thank you for accepting this interview, which is specially arranged for the journal Advanced Photonics. It is a photonics journal but we do not have to confine the topics to photonics. We can freely talk about anything related to mathematics, physics, and other things you like. The editors do hope that this interview can benefit readers in other disciplines.

Now Berry phase is almost everywhere in different branches of physics, chemistry, and many other subjects. It becomes impossible to overstate its importance. But there is something unfortunate about this: Michael Berry has been simplified and stereotyped as a phase. While, the fact is, you have absolutely original contributions to many fields of physics. It is unknown to many that you have also seminal contributions to mathematics, and moreover you have educated strong students who contribute significantly to physics and mathematics too. For example, your PhD student Jonathan Keating is now Sedleian Professor of Mathematics at Oxford University, a fellow of the Royal Society, and has been president of the London Mathematical Society. My first question is: why didn't you choose mathematics at the very beginning?

Michael Berry: Because I knew nothing about it. When I started as a graduate student, I was largely ignorant of mathematics. I knew I liked theoretical physics and I liked the ideas, but I wasn't very

*Address all correspondence to Wei Liu, wei.liu.pku@gmail.com

(C) The Authors. Published by SPIE and CLP under a Creative Commons Attribution 4.0 Unported License. Distribution or reproduction of this work in whole or in part requires full attribution of the original publication, including its DOI. [DOI: 10.1117/1 .AP.4.1.010503] knowledgeable about mathematics and it wasn't my initial enthusiasm. I discovered, while I was a graduate student, that I could do calculations and I liked some of the concepts in mathematics and found them natural. But until now I've almost never attended an advanced mathematics course. I still enjoy mathematical ideas, mostly when they have some connections with physics. (I don't mean experimental physics; that's not my main activity, though I've done a few experiments myself.)

Wei Liu: Then here comes a related question about your work style: you are a self-constructed intellectual soliton and you work more like a mathematician than a physicist. To explain this, here I have two quotations, one from the Fields Medalist Alain Connes and the other from the inaugural Abel Laureate and also Fields Medalist Jean-Pierre Serre. Connes said, "In general mathematicians tend to behave like fermions, i.e., avoid working in areas which are too trendy, whereas physicists behave a lot more like bosons, which coalesce in large packs and are often over-selling their doings, an attitude which mathematicians despise." Jean-Pierre Serre said, "The best ideas in maths are personal ones, and I am pretty sure about it." Would you please comment on what they said? To what extent can we say that the best ideas in physics must be personal?

Michael Berry: No, not really. The point about fermions is that they avoid each other. I don't avoid other scientists. I enjoy discussing with them, and as you can see from my papers I have occasional collaborators, though fewer than other physicists. I think science is essentially a collective activity, even among people who think they are solitary. They're not. We all build on ideas from other people, so that's my comment on Alain Connes, whom I know and respect.

I do not know Jean-Pierre Serre, but think I understand what he means, and I don't quite agree. We all feel personally engaged in physics about things that we discover. But within a few years, somebody else will probably have the same idea. Our apparently individual science is part of a developing collective understanding. It's a communal thing. I think science is the closest that we have to what in science fiction is called a group mind, in the sense that the huge advances that have come over the last centuries have been because we build (consciously or unconsciously) on what our colleagues and predecessors have done.

And I don't despise trending topics, as Alain Connes says. I am just not so inclined myself to work on subjects which are very popular: topological insulators, quantum Hall effect, high-temperature superconductors, string theory.... These are scientifically valid and interesting topics and it's good that people are working on them. Probably too many work on string theory but this is a minor issue-not the fault of creators of that subject. It is simply that my choices are a little bit different. Maybe I'm too lazy to read too much of the literature. I do read quite a bit actually, but not to systematically scour the literature to find every paper on something I'm working on. Occasionally that's a mistake: I discovered, quite recently, that there is already a substantial literature I was unaware of, on something I'm thinking about. 
Wei Liu: You have a kind of cruel comment on many topics. You never say it in public, but sometimes say it in private. The comment is: "this topic is interesting, but not fundamental." So I want to know your standard by which to judge what is fundamental and what is not.

Michael Berry: This is not a cruel comment. There's a great deal of physics, including much that I do, that isn't fundamental. I have had a few ideas in my life that in some sense might be considered fundamental, though not in the sense of Dirac, or Maxwell, or Galileo.... If one develops ideas in a pretty way and which has elegance, then this is valid. I'm frequently a referee and, depending on the journal, often say, "this is valid work; the calculations are correct. But it's really more suitable for a pedagogical journal than a journal for primary research." This isn't cruel; people might interpret it so but it is not. I'm just trying to be honest and it applies to much of my own work too. I have published quite a lot in European Journal of Physics, which is a journal of advanced pedagogy. I have published some work there which, in retrospect, others might have sent to (for example) Physical Review Letters. This is not a major concern for me.

Wei Liu: My feeling is that for something to be appealing to you, it would better to have a clear mathematical structure, and then there is a better chance for you to judge it as fundamental. Is this correct? Michael Berry: Probably, yes.

Wei Liu: So you like mathematical structures.

Michael Berry: Well, I think this is how physics is expressed, whether people realize it or not. The idea goes back to Galileo: the language of science is mathematical. He was thinking of geometry, but it's of course wider than that. And there's a reason for it which I've written about. Eugene Wigner had this famous phrase "the unreasonable effectiveness of mathematics in the natural sciences," because he was surprised, as we all are, when we suddenly find that some mathematics, developed some time ago, completely abstractly, fits directly some physics we're trying to understand. But I don't agree with him. It is of course a wonderful thing, but it isn't unreasonable at all. We want to understand aspects of the universe which haven't been understood before. However, we understand only those things we are capable of conceptualizing. And what are the most sophisticated conceptual structures that we humans have developed? Mathematical structures. Sometimes the mathematics exists already and sometimes it doesn't exist, and has to be created. I've encountered examples of both kinds; that's a detail. As humans, we are limited; we've been doing science for a mere few hundred years.

By the way, when people speak about the physics of everything, that's an expression of their enthusiasm, rather than anything that I take literally. We know a bit more than dogs. Dogs can't do quantum calculations and we can, but there are probably other things as remote from our understanding as ours is from that of dogs. We have learned to work together as a species and this collectivity enables us to make faster and deeper progress. But still we're limited. (It was pointed out to me that there at least one other sophisticated conceptual framework that human beings have developed that is as elaborate and rich as mathematics, namely music. I take that comment seriously but it doesn't affect my view about mathematics and physics.)

So I am disagreeing with Wigner. I expressed this disagreement when I gave the Wigner Lecture in the Oak Ridge Laboratory at Tennessee several years ago. I thought there would be a riot and I would be thrown out, but neither happened.

Wei Liu: You have a kind of humble background. Your mother was a dressmaker and father a taxi driver. You graduated from University of Exeter and University of St Andrews which are not the most prestigious ones. In my mind, because of this you don't like superficially more prestigious things. For example, last time you visited Changsha, you told me you like narrow streets with people crossing over here and there, which are more similar to the places where you grew up. I have a feeling that you simply do not like superficially more magnificent places. I want to know if this side of your character is related to the fact that you turned down the offer from Philp Anderson to join him in Princeton University.

Michael Berry: It wasn't Philip Anderson's offer but he knew about it. The suggestion originated with Martin Kruskal. Princeton is a wonderful place and was extremely kind to me; and there have been possibilities elsewhere. It's just that, when I thought objectively about the conditions of my daily scientific life and how I would live, in every case I came down in favor of staying in Bristol; it suits my scientific style.

There is something else. I know that my American colleagues spend a long time filing their income tax. In the UK, it takes me about 30 minutes every year. This is an example of something more general. If I had been in the US, I would have had to be much more of a financial manager than in the UK. Now it's a little bit different. In the UK, people younger than me who are not retired also have to be more financially adept than previously. So this difference has diminished; but it was a real difference then. We also had to think whether we wanted our children to be brought up in the US, rather than in the UK. I have nothing against the US and I love it. It's going through a slightly uncomfortable period at the moment but it's a wonderful place. I have many good friends there and I enjoy visiting magnificent landscapes and some of the beautiful campuses, but that's different from deciding to spend your life there.

Wei Liu: I would just tell you my feeling, which might be incorrect. It appears to me, there are two seemingly contradictory sides of your character. In everyday life, you like ordinary things, for example, you like narrow streets with people crossing over here and there. But for research, I describe you as an extreme elite. For example, you told me that science is not democracy and it is decided by the very best. These two sides seem to be contradictory and how can you reconcile them? Or they are just two sides of the same coin?

Michael Berry: Elite is not the same as elitist. It is democratic, in the sense that people from any background can and should be encouraged to do science; the more diversity the better. Nevertheless, as with other activities, such as being an accomplished musician or being an accomplished sports person, it's the elite who are respected. This is natural. But there are other subjects I enjoy just like any other human. I like cooking. I was spending some time just before this interview deciding what I should cook tonight. I had suggested to my wife that we go to a restaurant and she said no, because she's still a bit concerned about Covid in public places. I've just done some shopping and will cook tonight. I enjoy walking and looking around as I walk, or sometimes (in non-Covid circumstances) sitting in a cafe and just watching people or talking to people I encounter. It is perfectly normal. So I don't see any contradiction at all. Moreover, a major aspect of my science is demonstrating how abstract ideas often underlie our explanations of everyday phenomena, especially in optics (rainbows, the sun sparkling on the sea...); I call this "the arcane in the mundane."

Wei Liu: The next question is about your way of thinking. Your scientific hero Paul Dirac told Abdus Salam that his way of thinking is geometric rather than algebraic. You were quite surprised at the beginning by this. I want to know your way of thinking. Is it more algebraic than geometric, despite the fact that many people in Bristol have or had a geometric pictorial way of thinking? 
Michael Berry: Like many young theoretical physicists, I started out being very algebraic. I still do some long calculations-pages and pages of algebra (though uncomfortably aware of what Louis Armstrong is reputed to have said, "You can play lots of notes, but it is not music.”). But I soon realized that I am more naturally a visual person. This realization came partly because there was a visual/ pictorial tradition in Bristol physics. I'm sure it is not the only place but it's where I was, and I learned from listening to my colleagues that by making pictures I could understand things much better. You mention Dirac, and I note that he said that he was a visual person. Einstein said the same. You don't see pictures in their papers. It's very interesting. I don't doubt them but I asked Graham Farmelo, who wrote the major biography of Dirac: "You looked at Dirac's papers and his archive. Did you see lots of drawings and calculations hidden away?" Farmelo said he didn't. And when Dirac was at school in Bristol, he said one of his favorite subjects was technical drawing; there are some pictures from those days which recently came to light. What people say isn't always reflected in what they do publicly or even privately, but I don't doubt that Dirac had images in his head. Nowadays, it's much easier to make pictures; we have software that can do it. Even before software I made all my own illustrations for my papers, using pens, stencils, and drawing instruments. Nowadays we recognize more strongly the value of visual representation.

But we haven't yet recognized the value of auditory representation, and I started something along these lines with my occasional collaborator Pragya Shukla. We are calling it "Earmath": representing mathematics in sound. We have ears as well as eyes, and hearing is different from seeing. It's interesting how easy it is to produce qualitatively new sounds that no human has heard-representing, for example, random matrix eigenvalues, or their characteristic polynomials. At the moment this is a bit of a curiosity. As we wrote in a paper, whether it has scientific value "remains to be seen, or rather, heard."

Wei Liu: Well, you're very interested in and have made seminal contributions to the field of divergent series and asymptotics. I can't imagine how a person can be mainly visual and at the same time extremely interested in divergent series. How can divergent series be visualized?

Michael Berry: I regard divergent series as deeply connected with some philosophical matters, because divergent series are intimately related to the way in which different levels of descriptions arise (in physics, that is-I'm not talking about understanding life in terms of the Schrödinger equation). If you go from geometric optics to wave optics, or quantum to classical, the series you get always diverge. They must do so and there's a reason, related to the fact that these levels of description are very different from each other. If series converged, it would imply that quantum equals classical, plus a correction in $\hbar$, plus $\hbar$ squared, etc., and that isn't true. The limits are very singular, and one of the signals of a singular limit is that an associated series, that would connect one level with another, is divergent.

In different areas this requires mathematics, different in different areas, which sometimes has been developed and sometimes has not. Turbulence, for example, is the limit of fluid mechanics as the viscosity goes to zero. That's a singular limit, because if you just switch off the viscosity completely, and use the Euler equations, you don't have turbulence. As viscosity gets smaller, dissipation diminishes too, but it's distributed on a fractal set. That's completely different from what you get if you just simply write the equation without viscosity at all. This is just one example of qualitatively distinct science in the asymptotic borderland between theories. It is relevant when you come to consider the types of series that you encounter in describing the connections between levels, and why such series must diverge
Coming to your question: there is much visual material associated with divergent series. You show the series, first of all converging and then diverging. It is very vivid if you represent it pictorially. And in the method of stationary phase in the complex plane, for example, there are structures that become much clearer if you draw them: connections between different saddle points, Stokes lines between them, and so on.

In a major area of optics, namely geometric optics, the singularities are caustics, and caustics are where the series which are supposed to connect wave optics and ray optics are most strongly divergent. Caustics is a very pictorial subject. Just recently I have explored how caustics are central to the description of how you see your reflection distorted and topologically disrupted when looking at curved fairground mirrors, shiny curved metal reflections from cars, or metal spoons. These distorted reflections are everywhere, and I am explaining something actually seen, so of course it is visual. And connecting this with the deeper level of wave physics involves divergent series in an essential way. These ideas are very general.

By the way, going back to this question you asked about visual, when I started having independent ideas in physics, these were about semiclassical quantum mechanics. But I quickly realized that many of the problems are the same in optics, and in optics you have the advantage that you can see the phenomena you are thinking about. This is a different aspect of the visual approach: not that you are using pictures to explain mathematical connections, but that you're describing things you see with your eyes.

Wei Liu: So you believe you are more geometric than algebraic? Michael Berry: No, I wouldn't say that. If you look at my recent papers, you see algebraic calculations as well as physics. And "not more" doesn't mean less; I just don't know how to compare. Here is a story about Stokes (one of my intellectual heroes); I'm not sure if I get the details right. Stokes was a serious person; only once in his life was he heard to laugh. Why? It was at a dinner, where he was sitting next to a young woman, and it was her question that provoked him to burst into laughter. In those days, it was customary after dinner for all the women go to a different room to discuss (I don't know what), and the men to smoke and talk (I also don't know what about). The women gathered around the young woman and asked "What did you say to Sir George to make him laugh so?" "I asked him what he did, and he said, 'I'm a mathematician,' so I asked him: 'Do you prefer algebra or geometry?' He found this amusing."

Wei Liu: I have tracked your publications, and it appears to me that you got interested in C. V. Raman's works about sound and light at a very early stage of your career. Is this related to that your father stayed in India for a period of time? Besides Raman's works, you also like Indian food and culture.

Michael Berry: No connection. When I started my PhD I had no idea what problem I would work on and I asked my supervisor Robert Dingle; he had been developing fundamental ideas on divergent series (which I extended much later). He gave me a list of problems, where "My techniques might be helpful." The one I chose concerned the diffraction of light by ultrasound; basically an interaction of one kind of wave with another. It turned out that his mathematical techniques didn't help, but I did my $\mathrm{PhD}$ anyway. Some of the main papers on the subject were by Raman and his colleagues in Bangalore. At that time I had a wife and a baby and very little money. To earn some, I managed the library of theoretical physics in St Andrews. As the journals came in, I would scan them briefly. In almost every issue of the Proceedings of the Indian Academy of Science, there was a paper by Raman. So that was another connection. Only much later (in 1976, when I first visited India) 
did I learn about his brilliant nephew Pancharatnam and other members of his family, strengthening these connections. But I got interested in Raman's works very early because of my $\mathrm{PhD}$ thesis and the work that he had done with colleagues such as Nagendra Nath in the 1930s. This had nothing had to do with my father and his time in India, also in the 1930s.

Wei Liu: In another interview, you mentioned that you believe the Indian religion of many gods is healthier than the other one-God religion. Some people believe that the grand unified theory of everything, such as the string theory, is psychologically the same as the one-God religion. Can I infer from your comments on the Indian religion that you do not like the grand unified theory.

Michael Berry: It is not I don't like it. It is just a term. Any unification in physics is wonderful. The theory that would unify gravity with the others would be an enormous advance. But I don't think it would be the end of physics. That's all. I'm not in any way disparaging. And I don't think this has any special connection with Hinduism, one God or (in the superficial interpretation) many gods. My remark was not entirely serious. I'm not religious, certainly not in the sense of following an organized religion. So, my comment about Hinduism is simply that if forced to choose, it would be a religion with more gods. I certainly don't disparage grand unified theory. It's just a name, and that might mislead people into thinking it's the theory of everything.

And actually there's another reason. Even if there were a theory of everything (which I regard as impossible because of our limited intellectual capacity, as I've said), this wouldn't be the end of physics, because as Philip Anderson pointed out, and I've emphasized more generally, there's a different kind of fundamentalness: finding new things in old things. There's a story in one of the novels by Ian McEwen: a string theorist's wife discovers that he's been seeing another woman, and he tries to reassure her: "Don't worry, dear, I can explain everything." This is a perfect illustration of the fact that you can have a theory which in some abstract sense encompasses everything but doesn't help you to explain any particular thing. Dirac remarked that his equation explains all of chemistry; but it doesn't help a theoretical chemist wanting to understand the properties of a particular molecule. It's a matter of levels; it's just as fundamental to find, in the theories we already have, concepts that were not appreciated just by looking at the formalism. This was described in a beautiful way by Ian Percival, another physicist I admire (he was very helpful in the early days, by convincing me that there would be the subject we later called quantum chaos). He said that understanding the formalism is one thing, but understanding the solutions of the formalism is quite a different thing. This different kind of fundamentalness has been very influential for me.

Wei Liu: Now we come back to your geometric phase work. As far as I know, it's a byproduct of your some other research activities. Is this true?

Michael Berry: There's a sense in which everything is a byproduct of everything else, and then of course, it can take over one's life. For me, the geometric phase came via aspects of quantum chaos: understanding degeneracies. I had discovered something (actually rediscovered, as I soon learned): if you have some quantum system, depending on some parameters, the simplest case being a real symmetric matrix, and you cycle the parameters, then there will be a sign change if the cycle includes a degeneracy (where the energy of the state you are transporting coincides with the energy of another one). In a talk about this, I said, "This doesn't apply if you have a magnetic field; you need more parameters and the concept of enclosing is different." Ronald Fox responded, "What happens to the sign change then?" I replied, "I suppose there'll be a phase change." I went home and thought about it for several weeks, and talked about it with my colleague John Hannay, and then the geometric phase came to me.

But I learned afterwards that there were many anticipations of the phase, not exactly the same but with many similar ideas. I already mentioned Pancharatnam, but he wasn't the only one. Different people reached related insights in polarization optics, radio wave propagation, chemical reactions... Because the phase is connected with so many different areas of science, it could have emerged as a byproduct of different thoughts in different areas. In my case it happened to be quantum chaos. This is a subtle matter, and when I give lectures now on geometric phase I often include the timeline, to acknowledge all the predecessors I was completely ignorant of when I wrote my paper in 1983. This is true generally; if you look at Einstein's special relativity, Poincaré and Lorentz came extremely close shortly before him. Somebody once said to Dirac how surprising it is that discoveries occur at almost the same time to different people, and it could be that something is already "in the air"; Dirac replied "Or maybe in the water."

Wei Liu: The Berry phase work is certainly your most famous one. But surprisingly you told me that it is not your favorite one. As I try to understand why it is so, I come to believe that there must be some personal psychological reasons. Your colleague Sandu Popescu said, "When he (Michael Berry) sees everybody going in one direction, he goes in another." I have a strange feeling that if many people like something, you would not like it anymore. Is this one of the reasons why you don't believe the Berry phase work is your favorite?

Michael Berry: No. This is a misunderstanding. I don't dislike something just because many other people like it. I already mentioned that there are fashionable subjects and I have a great respect for them. I just choose not to move in those directions. I'm delighted by the success of geometric phase and I don't dislike it at all. I see the significance of it, largely because of the ways other people have developed it. I have six children, and if someone asks me who is my favorite, I would never answer such a question. My children are different, and I love them all for their different aspects.

Nevertheless, I mention something that gave me immense pleasure. It relates to divergent series; understanding something called the Stokes phenomenon. It pleased me because it was a discovery in mathematics, which as I have said isn't my main scientific habitat. To understand something left open from Stokes 150 years before (the smoothing of a discontinuity) was very, very satisfying - a pleasant little thing. As you said, this is a psychological matter; I certainly didn't study the Stokes phenomenon because I don't like things that other people like. Let's not propagate the misunderstanding.

Wei Liu: The next question might be related. It is striking for me that you have never published with the American Physical Society, that is, you have never published in the Physical Review journals. What's the reason behind that?

Michael Berry: No particular reason. I have published in a number of different American journals: Annals of Physics, Journal of the Optical Society of America, Journal of the Acoustical Society of America, PNAS, Physics Today, and also Scientific American. But I am perfectly happy with the local, i.e., UK, journals, mainly those published by the Institute of Physics, which is the second biggest physics publisher in the world. IoP Publishing happens to be based in Bristol, but I don't think that's significant. I don't go to their offices very often; they are in a different part of town from the university. I have found IoP very reliable and they have high standards.

When I agreed to be the editor of Proceedings of Royal Society, section A, a post I held for six years, I was very proud. It's almost 
the world's oldest scientific journal. Many eminent scientists (Dirac, Maxwell, Rayleigh, Rutherford, and others) published there; I might have published more papers in Proc R. Soc A (including some of my best) than anybody else. The Royal Society had very high standards. Many years earlier, in connection with a paper in Philosophical Transactions of the Royal Society, they telephoned to ask whether a symbol, a subscript in one of my handwritten equations, was the number "zero" or the letter "o." I was very impressed by that, but by the time I was editor, their methods of production weren't quite as good as those of IoP, so I insisted that some of their production people visit Bristol to talk to the IoP, to learn how they did things, and possibly make improvements in London. I'm not quite so happy now with some of IoP's online procedures, proof corrections, and other software, but still I like publishing with them. Certainly the quality of refereeing is very good, and I have learned much from reports on my papers, even (especially) when these are critical.

Wei Liu: You simply do not care about the rankings of journals. Is this a manifestation of your philosophy that science is not democracy; it is decided by the very best?

Michael Berry: I am not sure it is related. People who design journal ranking metrics are very emphatic that these metrics provide information useful for editors, but should never be used to assess individuals. I strongly agree, and would go further: using journal citation statistics, or rankings, to assess an individual, is an intellectual crime analogous to racism (though of course far less serious), because it amounts to judging individuals by a stereotype of the group to which they are considered to belong, rather than by their individual work. In making this remark I don't expect to have any influence, but there are signs that this fashion for journal rankings is beginning to diminish (differently in different parts of the world). This is not a main concern for me; I publish in the journals I like and know.

Wei Liu: Now we go back to Pancharatnam. Largely due to your expositions, Pancharatnam's work about geometric phase became well known among the general researchers. In your paper titled "Pancharatnam, virtuoso of the Poincaré sphere: an appreciation," you discussed mainly three papers of Pancharatnam, covering geometric phase, mirage, and Pancharatnam phenomenon. Something strange to me is that now so many people are working in the field of nonHermitian physics, but very few of them know Pancharatnam phenomenon. Do you know what the reason is behind this?

Michael Berry: I do not know. You ask me why people don't know something; probably you should ask them. In a sense people might know Pancharatnam's phenomenon, but not the name of it. I gave it that name out of respect for Pancharatnam, after thinking mathematically about an optical phenomenon he described.

It concerns a system depending on parameters and with two eigenvalues. This tells you about the time development of something: you get exponentials that depend on the time, and they interfere with one another, and you observe beats. If they are degenerate, then in Hermitian physics there are still two separate eigenstates, and the beat frequency is zero. But if the system is non-Hermitian (e.g., if there is dissipation or gain) the two eigenvectors coincide as well as the eigenvalues. What happens, Pancharatnam asked, if you send in a beam of light with the wrong (i.e., orthogonal) polarization for the particular parameters of which there are degeneracies? Voigt, who in the 1900s did a lot of pioneering work on non-Hermitian physics, thought the light would be reflected. No. Pancharatnam showed that the polarization slowly evolves into the correct eigenvector. This is because, although there is one exponential instead of two, there are still two states; one of them is multiplied by $t$ (or $x$ if the evolution is in space).
I called this the ghost of the departed eigenvector, and I named it Pancharatnam's phenomenon because it explains what he did. He understood it in his own way, not in the algebraic way, but this doesn't matter. It turned out to explain a number of other things, including experiments Anton Zeilinger had done. I was very pleased to find Pancharatnam's paper and I thought his discovery deserved a name. Some names catch on and some don't; I do not feel very strongly about this. Pancharatnam was a most original person, who died tragically young. His widow still lives in Oxford and I have met her. But I never met Pancharatnam and although I knew about Raman I never met him either. In the 1960s I had no connection with India and had no reason to know about Pancharatnam, because I didn't know much about polarized light.

I don't think my review that you mention is responsible for the posthumous appreciation of Pancharatnam. It's much more the fact that, when I visited India for the second time and spoke about the geometric phase in 1986, Rajaram Nityananda and also Sivaraj Ramaseshan told me about the work of Pancharatnam and which they had remembered and just written about in Current Science. But my paper, that I wrote on the plane returning from India, "The adiabatic phase and Pancharatnam's phase for polarized light" may have done more to revive his reputation, as well as my review in Current Science.

Wei Liu: Have you discussed this Pancharatnam phenomenon with your friend Vladimir Arnold? I think he would be very interested, because this belongs to his beloved field of partial differential equations. Michael Berry: He would have liked it, but I didn't discuss with him and we didn't discuss non-Hermitian operators at all. I met him in 1990, when he came to Bristol. He stayed in my house for a week and we discussed many things. But at that point I had not started thinking seriously about non-Hermitian operators. He was concerned mainly with Hermitian operators in Hamiltonian systems (he had some work on dissipation but nothing quantum). So I don't think he knew or thought much about non-Hermitian degeneracies, which is the heart of my own interest in the subject.

Wei Liu: Have you discussed this phenomenon with Carl Bender, who is now famous for PT symmetry?

Michael Berry: I don't remember if I did. He might have heard me speak about it, but specifically this Pancharatnam phenomenon, concerning the non-Hermitian degenerate state?-No, I don't think I discussed it with him. Whether he would be interested in it, I am not sure. He certainly knew about the degeneracies, more generally as well as in the context of PT symmetry. PT has been very popular but it's a small part of non-Hermitian mathematics and physics. Of course degeneracies do occur in PT systems, resulting in what Carl Bender calls PT symmetry breaking. There is nothing particularly different about such degeneracies being PT. So I don't think we've specifically discussed this.

Wei Liu: Here we come to your contributions to singularities, including singularities of intensity, caustics; singularities of phase, vortices; and singularities of polarization. Who influenced you most on your singularity works? Is that Vladimir Arnold and René Thom?

Michael Berry: Neither. It was before interactions with them. Their work was a revelation later, when I realized that it was in a sense the completion of one aspect of the subject that I will talk about in a moment. I was interested in caustics before, from lectures I heard in 1964, when I was a graduate student, lectures by J. L. Synge, a brilliant relativist from Dublin. The first lecture course I gave, when I was a graduate student (again to earn some money: my supervisor was sensitive to the fact that I had a young child), was on general relativity. 
This was very difficult for me; as I told you, I didn't know much mathematics then. While preparing the course, I read many books, and the ones that really impressed me were by Synge. My supervisor, Professor Dingle, told me that Synge would be giving some lectures in London, and I attended them. But the lectures weren't about relativity at all. They were about the Hamiltonian theory of rays and waves, and caustics played a role (describing water waves scattered by an island). I realized immediately that caustics would be central in combining two subjects I was beginning to glimpse as areas for future study: the mathematical asymptotics that Dingle was developing, and understanding the connection between classical and quantum. Synge's lectures had a big influence on me. So I like caustics and soon discovered that you see them not only in quantum physics but also in optics, for example on the bottom of swimming pools.

When I learned about Thom and Arnold, which was 10 years later, what was important is that they described the forms of caustics that occur naturally, which is the exact opposite of what you have in optical instruments. In optical instruments you artificially seek to create caustics that are points: nongeneric, unstable. The perfect focus is an example; anything else was what in optics is called an aberration. The new mathematics, called catastrophe theory, enabled concrete statements about caustics that occur naturally. In Bristol we extensively developed "catastrophe optics" as a distinct area of physics, culminating in my long review with Colin Upstill in 1980, and the masterly book by John Nye 20 years later. Already in the 1960s, before I knew about Thom and Arnold and catastrophes, I had realized that caustics were the singularities of ray optics, places where wave optics is most dramatically apparent: it smooths the singularities in characteristic ways, and decorates them by interference.

Wei Liu: After caustics, the next question is about phase singularity and its difference from orbital angular momentum. Do you think phase singularity is more fundamental and generic than orbital angular momentum? Phase singularities do not necessarily produce non-zero orbital angular momentum, while I believe when there is orbital angular momentum, phase singularities have to be present somewhere.

Michael Berry: Phase singularities are certainly generic, but I cannot say if they are more fundamental. They describe different aspects, related in some ways, and not others. My own inclination, according to my general scientific philosophy, is towards understanding the singularities, because this is the way in which one regime of physics is related to another. Phase singularities (a.k.a. optical vortices, wave dislocations, or nodal sets) are the singularities of wave optics in the sense that caustics are the singularities of ray optics; there is complementarity, a perspective I like.

Orbital angular momentum is very important and fundamental, even though the connection with phase singularity is more an overlap than an identity. I refer to the fact that orbital angular momentum isn't directly associated with the zeros, because there's no intensity there. But it's associated with aspects of the wave away from the zeros, and the zeros sometimes can organize orbital angular momentum. This is especially true in many applications, which is why this myth persists about the connection being inevitable. If you are considering eigenstates of orbital angular momentum, then the two concepts are connected. But you can have superpositions of eigenstates and there, as I showed with examples in the 1990s, you can have distributions of singularities unrelated to the orbital angular momentum, which is an integral over the whole beam.

Wei Liu: You told me Les Allen discussed with you on a train about his work on orbital angular momentum before it was published in Physical Review A, which is now a very famous paper. You did not recognize the significance of his work at the very beginning, and you have just explained why. What surprises me is that Les Allen and others had also failed to recognize the significance of your work with Nye about phase singularity, as he did not cite your paper in his Physical Review A paper anyway. Can you guess the reason why most researchers working on orbital angular momentum have failed to appreciate the significance of phase singularities?

Michael Berry: People have different interests, and it's taken a long time for the singularity point of view, if I can put it that way, to become popular. It is still not as popular as, for example, mechanical analogies like angular momentum. You asked me about the psychology of my fellow physicists, who might appreciate one and not the other; you should ask them. My guess is that the singularity approach is still thought of as something rather slightly eccentric, off the mainstream, although it is getting more popular now. Sometimes it takes time for ideas to penetrate and become popular. It hasn't fully happened with singularities, to the extent that I have hoped; I'm not complaining.

Wei Liu: The next question is about polarization singularity. When you explain geometric phase, you would refer to Hamilton's diabolical point, conical refraction and the associated half-turn of the polarization. Why don't you interpret this half-turn from the perspective of singular optics? You always interpret this from the perspective of geometric phase.

Michael Berry: I don't. The papers I wrote on conical refraction, starting in 1994, all emphasized the phenomenon as a singularity. At the same time, it illustrates geometric phase in a simple way-something I came to appreciate a little later. And it's a nice way to give a historical perspective because it's the first example known to me of a geometric phase in physics. When I give talks, I emphasize the connection with conical intersections, and I note that Hamilton understood these (implicitly) as singularities in the Fresnel wave surface.

Wei Liu: You actually wrote a paper with Mark Dennis in 2003, titled "The optical singularities of birefringent dichroic chiral crystals," extending the original work on Hamilton's diabolical point and conical refraction. It has been extended into crystals with chirality and loss, and then there is non-Hermitian degeneracy. So you remember that work with Mark Dennis?

Michael Berry: I do remember it and I'm very proud of it. But that concerned the formalism of crystal optics, and I didn't quite think of it as extending Hamilton; conical refraction is a particular phenomenon. You can see some of these structures in crystals by sending a beam in, but this was not a major part of the work with Mark Dennis. I developed the theory of conical refraction a year or so after the paper with Mark Dennis. And I worked on it with my student Mike Jeffrey, experimentally and also extending my paper to include chirality and dissipation, where some quite different phenomena occur; this culminated in our review for Progress in Optics.

The paper with Mark Dennis recreated crystal optics based on singularities, as you know. We dedicated the paper to Sivaraj Ramaseshan and John Nye who were both 80 at about that time. This was something we were very happy to do, because John Nye wrote his highly influential Physical Properties of Crystals, containing much more than optics (for example elasticity and symmetry groups), and Ramaseshan had written (with Ramachandran) the classic Handbuch der Physik article on crystal optics in the 1960s. I knew both, and respected them as people as well as scientists. The paper with Dennis considered the space of possible crystals in optics, based on the dielectric tensor (also the magnetic, but bianisotropy is something that came a little later). The dielectric tensor is a $3 \times 3$ matrix. If it's simply birefringent, it's a real symmetric matrix; if chiral, it's complex Hermitian; if it has loss, it is 
non-Hermitian. There are many parameters; how do you navigate in this enormous space? The singularities help greatly, by characterizing all the different things that can happen, qualitatively and quantitatively. That was our perspective: singularities (three different kinds) as organizing concepts.

Wei Liu: You did not discuss geometric phase in that paper with Dennis. Michael Berry: We did talk about it because we wanted to define the local wavevector, but we didn't discuss changing parameters, even though of course I knew the work of Pancharatnam by then and had already written about it. The full understanding of geometric phase in a spatially varying field came only relatively recently, in work by Dennis, with Konstantin Bliokh and Miguel Alonso, not me.

Already in the mid-1990s I had worked with Susanne Klein, making stacks of crystal plates, enabling us to demonstrate Pancharatnam's geometric phase for circuits on the Poincaré sphere. In the Raman Institute, Rajendra Bhandari had written similar papers. His circuits all involved arcs of great circles, but we wanted to understand how to make general circuits, by using combinations of quarter-wave plates, half-wave plates, polarizers, etc., and carry out demonstration experiments.

Wei Liu: You have seminal contributions to geometric phase and you have seminal contributions to polarization singularities, but you rarely discuss them in the same paper. Is that true?

Michael Berry: Well, no, it's not true. In my original geometric phase paper I emphasized their connection with degeneracies, which are the singularities of the spectrum, and the sources of abstract monopoles.

Wei Liu: I mean connections between geometric phase and polarization singularity, not general singularities.

Michael Berry: My 1987 paper was precisely about the connection between Pancharatnam's phase in crystal optics and the geometric phase in quantum mechanics. But you are right: there was no emphasis on the Nye-Hajnal polarization $C$ and $L$ line singularities. In a sense those phase changes are elementary: $\pi$ round a $C$ line and $2 \pi$ round an $L$ line.

Wei Liu: We are now close to the end of this interview. I know you like music such as jazz, you like philosophy, and you like cooking. You even write poems. You wrote one about the Stokes phenomenon that you love.

Michael Berry: That was my only poem.

Wei Liu: Here I have a quotation from Dirac about poems and I would like to know what you think about it. Dirac said, "Physics and poems are in opposition. In science you want to say something nobody knew before, in words which everyone can understand. In poetry you are bound to say something that everybody knows already in words that nobody can understand."

Michael Berry: I'm not sure Dirac was entirely serious. I am not somebody who usually understands poetry, but this isn't because I believe that poets deliberately or unconsciously write in ways that are obscure. It is that the level of abstraction and connections are often too abstruse for me to grasp— just as in some papers on pure mathematics, where the levels of abstraction are so general and assume so much that I don't understand. I'm not criticizing poets, and I don't agree with Dirac. There are different ways of hinting at connections that are not captured by physics and mathematics.
Wei Liu: In your recent book, you thanked your children and three wives. I can thus believe that there must be turbulent times during your life, but this was not reflected at all in your publication list shown on your wonderful website. How did you go through those turbulent periods?

Michael Berry: During troubled times in your life, doing science can be good therapy. When I was starting out in physics in my late 20s, my first wife and I were divorcing (we are good friends now), and doing physics enabled me to have some peace of mind during that difficult time. But now, thinking back, I would say that my domestic life has overall had very little effect on the physics I've done. There were times when I've thought they were more connected. This is a psychological matter, and one is unwise to pretend one understands oneself sufficiently to make reliable comments. Physics has been a constant, although my wives will tell you that there have been times when I've declared, after failing to understand something, that my days creating physics were over. But somehow new ideas came, and those episodes of disappointment faded.

Wei Liu: At the end, can you give young researchers some suggestions, especially for those in developing countries like India and China.

Michael Berry: There are plenty of role models. In India for example, Raman, and the astonishing mathematician Ramanujan (by the way, they came from the same area of Tamil Nadu, very interesting culturally). People often ask what they should work on. Successful scientists are to some extent role models, but physics has to come from inside. You might take a while to find your feet in physics (for me, it was several years), but it's ok to relax and enjoy exploring possibilities with determination and energy.

There is a subject I'm enthusiastic about but don't work on: quantum information theory. I think it's enormously important. If I were starting afresh, I might choose that area (even though it's fashionable!). It will change civilization, as mechanics, electromagnetism, and in the last century ordinary quantum mechanics, have changed civilization, in ways much more important than this or that king or queen or emperor or revolution or nationalism or whatever. Quantum information technology will be distinct from technologies that arose from previous quantum mechanics, because it involves the manipulation of individual quantum states. It is going to be hugely influential, in ways we can't predict.

Wei Liu: Professor Berry, this would be the end of this interview. Thank you very much for your suggestion and for all that you said in this interview.

Wei Liu obtained his BSc and PhD degrees, both in physics, from Peking University and Australian National University, respectively. He works mainly on Mie theory, and its interactions with symmetry, topology, and singularity.

Sir Michael Berry is a theoretical physicist at the University of Bristol, where he has been for more than twice as long as he has not. His research centers on the relations between physical theories at different levels of description (classical and quantum physics, ray optics and wave optics...). In addition to these deeply mathematical, often geometric, studies, he also delights in finding familiar phenomena illustrating deep concepts-the arcane in the mundane: rainbows, the sparkling of the sun on the sea, twinkling starlight, polarized light in the sky, tidal bores... For more information about Sir Michael Berry, please refer to https:// michaelberryphysics.wordpress.com. 